\title{
Effect of diet-induced weight loss on lipoprotein(a) levels in obese individuals with and without type 2 diabetes
}

\author{
Kirsten A. Berk ${ }^{1} \cdot$ Reyhana Yahya $^{1}$ - Adrie J. M. Verhoeven ${ }^{1} \cdot$ Jeanette Touw $^{1}$. \\ Frank P. Leijten ${ }^{1}$ - Elisabeth F. van Rossum ${ }^{1}$ • Vincent L. Wester ${ }^{1}$ - Mirjam A. Lips ${ }^{2}$. \\ Hanno Pijl ${ }^{2}$ - Reinier Timman ${ }^{3}$ - Gertraud Erhart ${ }^{4}$ - Florian Kronenberg ${ }^{4}$. \\ Jeanine E. Roeters van Lennep ${ }^{1}$ • Eric J. G. Sijbrands ${ }^{1} \cdot$ Monique T. Mulder $^{1}$
}

Received: 27 October 2016 / Accepted: 20 February 2017 /Published online: 6 April 2017

(C) The Author(s) 2017. This article is published with open access at Springerlink.com

\begin{abstract}
Aims/hypothesis Elevated levels of lipoprotein(a) [Lp(a)] are an independent risk factor for cardiovascular disease (CVD), particularly in individuals with type 2 diabetes. Although weight loss improves conventional risk factors for CVD in type 2 diabetes, the effects on $L p(a)$ are unknown and may influence the long-term outcome of CVD after diet-induced weight loss. The aim of this clinical study was to determine the effect of diet-induced weight loss on $\mathrm{Lp}$ (a) levels in obese individuals with type 2 diabetes.

Methods Plasma Lp(a) levels were determined by immunoturbidimetry in plasma obtained before and after 3-4 months of an energy-restricted diet in four independent study cohorts. The primary cohort consisted of 131 predominantly obese patients with type 2 diabetes (cohort 1), all participants of the Prevention Of Weight Regain in diabetes type 2 (POWER) trial. The secondary cohorts consisted of 30 obese patients with type 2 diabetes (cohort 2), 37 obese individuals without type 2 diabetes (cohort 3 ) and 26 obese
\end{abstract}

Kirsten A. Berk and Reyhana Yahya contributed equally to this study.

Monique T. Mulder

m.t.mulder@erasmusmc.nl

1 Department of Internal Medicine, Erasmus Medical Center, Office Ee800, PO Box 2040, 3000 CA Rotterdam, the Netherlands

2 Department of Internal Medicine, Leiden University Medical Center, Leiden, the Netherlands

3 Department of Psychiatry, Section of Medical Psychology and Psychotherapy, Erasmus Medical Center, Rotterdam, the Netherlands

4 Division of Genetic Epidemiology, Department of Medical Genetics, Molecular and Clinical Pharmacology, Medical University of Innsbruck, Innsbruck, Austria individuals without type 2 diabetes who underwent bariatric surgery (cohort 4).

Results In the primary cohort, the energy-restricted diet resulted in a weight loss of $9.9 \%$ (95\% CI 8.9, 10.8) and improved conventional CVD risk factors such as LDL-cholesterol levels. Lp(a) levels increased by $14.8 \mathrm{nmol} / 1$ (95\% CI 10.2, 20.6). In univariate analysis, the change in $\mathrm{Lp}$ (a) correlated with baseline $\mathrm{Lp}(\mathrm{a})$ levels $(r=0.38, p<0.001)$ and change in LDL-cholesterol $(r=0.19, p=0.033)$. In cohorts 2 and 3 , the weight loss of $8.5 \%$ (95\% CI $6.5,10.6)$ and $6.5 \%$ (95\% CI 5.7, 7.2) was accompanied by a median increase in $\mathrm{Lp}$ (a) of $13.5 \mathrm{nmol} / 1(95 \%$ CI $2.3,30.0)$ and $11.9 \mathrm{nmol} / 1$ (95\% CI 5.7, 19.0), respectively (all $p<0.05$ ). When cohorts 1-3 were combined, the diet-induced increase in $L p(a)$ correlated with weight loss $(r=0.178, p=0.012)$. In cohort 4 , no significant change in $\mathrm{Lp}(\mathrm{a})$ was found $(-7.0 \mathrm{nmol} / 1 ; 95 \%$ CI $-18.8,5.3)$ despite considerable weight loss $(14.0 \%$; $95 \%$ CI 12.2, 15.7).

Conclusions/interpretation Diet-induced weight loss was accompanied by an increase in Lp(a) levels in obese individuals with and without type 2 diabetes while conventional CVD risk factors for CVD improved. This increase in $L p(a)$ levels may potentially antagonise the beneficial cardiometabolic effects of diet-induced weight reduction.

Keywords Apolipoprotein(a) $\cdot$ Bariatric surgery $\cdot$ Diet . Lipoprotein(a) - Obesity $\cdot$ Type 2 diabetes $\cdot$ Weight loss
Abbreviations
AHEAD Action for Health in Diabetes
Apo(a) Apolipoprotein (a)
CVD Cardiovascular disease
HMW High molecular weight 


$\begin{array}{ll}\text { IQR } & \text { Interquartile range } \\ \text { KIV } & \text { Kringle-IV type } 2 \\ \text { LMW } & \text { Low molecular weight } \\ \text { Lp(a) } & \text { Lipoprotein (a) } \\ \text { MANOVA } & \text { Multivariate ANOVA } \\ \text { sLR11 } & \text { Soluble form of the LDL receptor relative with } \\ & \text { 11 ligand-binding repeats } \\ \text { VLCD } & \text { Very low calorie diet }\end{array}$

\section{Introduction}

Cardiovascular disease (CVD) is the main cause of morbidity and mortality in obese individuals with and without type 2 diabetes [1-3]. The risk of CVD in obese patients with type 2 diabetes has been attributed to age, smoking, hyperglycaemia, hypertension and dyslipidaemia [2]. Weight loss via lifestyle programmes, consisting of diet and physical activity, results in an improvement in conventional CVD risk factors and is first-line therapy to slow down the development of type 2 diabetes and the progression of its complications in overweight or obese individuals $[4,5]$.

Lipoprotein a $[\mathrm{Lp}(\mathrm{a})]$ is an independent risk factor for CVD [6-12]. Lp(a) consists of an LDL-like particle with an additional apolipoprotein (a) [Apo(a)] attached to it. Plasma Lp(a) concentrations vary highly between individuals and are largely genetically determined by the number of copies of kringle-IV type 2 (KIV-2) in the Apo(a) protein [Apo(a) isoform] [13-16]. A low number of copies of KIV-2, associated with elevated levels of $L p(a)$, has been shown to increase the risk of CVD [17]. A recent prospective population-based cohort of 56,367 participants showed a significantly higher contribution of $\mathrm{Lp}(\mathrm{a})$ levels to CVD and risk of myocardial infarction in patients with type 2 diabetes than in control participants without type 2 diabetes [18]. About $25 \%$ of the variance in $\mathrm{Lp}$ (a) levels has been attributed to lifestyle [19]. Weight loss in obese individuals has been reported to affect $\mathrm{Lp}$ (a) levels, but the results are controversial [20-23]. The effect of weight loss on plasma $L p(a)$ levels in type 2 diabetes has not yet been determined.

The aim of the current study was to determine the effect of diet-induced weight loss on $\mathrm{Lp}$ (a) levels in obese patients with type 2 diabetes. In order to confirm our findings we also examined the effect of weight loss on $L p(a)$ levels in three independent cohorts of obese patients with or without type 2 diabetes. As a secondary aim, we assessed the influence of Apo(a) isoforms on diet-induced changes in $\mathrm{Lp}(\mathrm{a})$ level in individuals with type 2 diabetes.

\section{Methods}

Participants and interventions The effect of weight loss was examined in four independent cohorts. The primary cohort (cohort $1, n=131$ ) consisted of overweight and obese individuals (BMI $>27 \mathrm{~kg} / \mathrm{m}^{2}, 93 \%$ obese) with type 2 diabetes who participated in the run-in phase of the Prevention Of Weight Regain (POWER) trial (trial registration no. NTR2264) [24]. This trial aimed to study long-term weight maintenance after the run-in diet phase. The sample size of 131 patients was sufficient to find a difference of $10.6 \mathrm{nmol} / 1$ $(5 \mathrm{mg} / \mathrm{dl})$ in $\mathrm{Lp}(\mathrm{a})$ level with a baseline-to-end correlation of 0.95 between the measurements, an $\alpha$ of 0.05 and a power of 0.90 . The diet started with 8 weeks of a diet very low in energy (very low calorie diet [VLCD]) of approximately $3000 \mathrm{~kJ}$ (750 kcal) per day, consisting of two meal replacements (Glucerna, Abbott Nutrition, Lake Forest, IL, USA) and a small dinner daily. Thereafter, energy intake was slowly increased up to approximately $5500 \mathrm{~kJ}(1300 \mathrm{kcal})$ per day (a low-energy diet) over 12 weeks. Some of the baseline characteristics and effect of the diet on body weight in cohort 1 have previously been reported [25].

Cohort $2(n=30)$ also consisted of overweight and obese patients ( $80 \%$ obese) with type 2 diabetes, who were recruited after the POWER trial had finished, to study the implementation of a VLCD for weight loss in type 2 diabetes. The participants underwent the same dietary intervention as the patients in the primary cohort. Cohorts 1 and 2 were both recruited from the outpatient diabetes clinic of the Erasmus Medical Center, Rotterdam, the Netherlands. To reduce risk of hypoglycaemia, doses of insulin and sulfonylurea derivates were lowered before the start of the diet but after baseline measurements had been made. During the diet, the insulin dose was regularly adjusted to achieve optimal glycaemic control. Metformin use was continued. Only two participants were taking glucagon-like peptide 1 (GLP-1) receptor agonist treatment, which was continued during the intervention period. Statin treatment was kept unchanged during the study.

Cohort 3 consisted of 37 obese individuals without type 2 diabetes, who were recruited at the Obesity Center 'Centrum voor Gezond Gewicht' of the Erasmus Medical Center. They underwent a 3 month dietary intervention consisting of a $2000 \mathrm{~kJ}(500 \mathrm{kcal})$ per day reduction in intake relative to baseline (low-energy diet), with macronutrient and micronutrient content in line with national dietary guidelines, while exercise was encouraged.

Cohort 4 consisted of 26 obese individuals without type 2 diabetes who underwent gastric banding $(n=10)$ or a gastric bypass procedure $(n=16)$. These participants were recruited at the Leiden University Medical Center, Leiden, the Netherlands. No specific diet was recommended beyond a staged meal progression during the first 3 months after surgery. Analyses were performed at baseline and 3 months after surgery.

The dietary intervention studies and $\mathrm{Lp}(\mathrm{a})$ analysis of previously collected clinical samples were approved by the Medical Ethics Committee of the Erasmus Medical Center 
(reference numbers MEC-2009-143, MEC-2014-090 and MEC 2016-604). The bariatric surgery study and use of the samples was approved by the Medical Ethics Committee of the Leiden University Medical Center (reference number MEC P08.215). All investigations were carried out in accordance with the principles of the Declaration of Helsinki (2008). All participants provided written informed consent.

Measurements Blood samples were obtained after an overnight fast and were stored at $-80^{\circ} \mathrm{C}$ until further analysis. Demographic variables were recorded, and weight, height and waist circumference (except for cohort 4) were measured. Ethnicity was expressed as white or non-white. $\mathrm{HbA}_{1 \mathrm{c}}$, fasting glucose, total cholesterol, LDL-cholesterol, HDL-cholesterol and triacylglycerol were measured using standard laboratory techniques.

Lp(a) measurement Plasma Lp(a) concentrations were measured using a particle-enhanced immunoturbidimetric assay, which was largely independent of Apo(a) KIV repeat number (Diagnostic System \#171399910930; DiaSys Diagnostic System, GmbH, Holzheim, Germany) [26]. Plasma samples were stored at $-80^{\circ} \mathrm{C}$ for $0.5-5$ years and thawed for the first time prior to this analysis. For each individual, levels at baseline and after intervention were measured in the same run. The detection limit of the assay was $6 \mathrm{nmol} / 1$, and the mean intra-assay variability was $2.8 \%$. Interference of triacylglycerol with $\mathrm{Lp}$ (a) measurements was minimal, as measured Lp(a) levels were less than $5 \%$ affected by the addition of plasma containing different concentrations of triacylglycerol (ranging from 0 to $12 \mathrm{mmol} / \mathrm{l}$ ) to plasma with a relatively high $\mathrm{Lp}$ (a) concentration (169 or $338 \mathrm{nmol} / \mathrm{l}$ ). Repeated sampling in 27 healthy control individuals at an interval of 2-6 months did not reveal significant differences in median Lp(a): $29.3 \mathrm{nmol} / \mathrm{l}$ (interquartile range [IQR] 17.5-87.8) vs $26.4 \mathrm{nmol} / \mathrm{l}$ (IQR 12.4-60.3), $p=0.087$, for day 0 and after 2-6 months, respectively.

In the primary cohort (cohort 1), the Apo(a) KIV repeat number was determined by immunoblotting, as previously described [27, 28]. When two distinct Apo(a) isoforms were present, the band representing the smaller isoform showed the strongest intensity in most cases and was used as a continuous variable. Apo(a) KIV repeat numbers were stratified in two groups as previously described [28]: low molecular weight (mass) (LMW) when at least one isoform with 22 or fewer KIV repeats was present, and high molecular weight (mass) (HMW) when only isoforms with more than 22 repeats were present.

Statistical analysis Normality of the data and homogeneity of variances were tested using the Shapiro-Wilk test and Levene's test. Variables were expressed as mean $\pm \mathrm{SD}$ or as median with IQR, and were tested for statistical significance using a two-sided paired-sample $t$ test or a Wilcoxon ranking test, depending on the normality of the data. Medians and 95\% CIs were calculated using ratio statistics, and median differences were analysed using a related-samples Hodges-Lehman test. Owing to the low numbers in cohorts 2, 3 and 4, in-depth analyses were performed only for cohort 1. We determined Spearman correlations of both baseline $\mathrm{Lp}$ (a) levels and change in $\mathrm{Lp}$ (a) with different variables of weight loss and glycaemic control.

Mann-Whitney U tests were used to analyse the difference in baseline $\mathrm{Lp}$ (a) levels between the LMW and HMW subgroups. Repeated-measurements multivariate ANOVA (MANOVA) analysis (on Blom-transformed outcome variables) was used to analyse the difference in change in Lp(a) between subgroups. SPSS version 21.0 (IBM, Armonk, NY, USA) and GraphPad Prism version 5 (GraphPad Software, La Jolla, CA, USA) were used for the statistical analyses.

\section{Results}

Effect of diet on obese patients with type 2 diabetes (cohort 1) The characteristics of the primary cohort (cohort 1) at baseline and after intervention are shown in Table 1. The 131 individuals were predominantly obese, as $93 \%$ had a BMI greater than $30 \mathrm{~kg} / \mathrm{m}^{2}$. The remainder had a BMI >27 and $\leq 30 \mathrm{~kg} / \mathrm{m}^{2}$. This cohort had a mixed ethnic background (56\% white, and 44\% non-white: South-Asian and African). Baseline Lp(a) levels correlated negatively with Apo(a) KIV repeat number $(r=-0.53, p<0.001)$, baseline weight $(r=-0.18, p=0.046), \mathrm{HbA}_{1 \mathrm{c}}(r=-0.20, p=0.022)$, fasting triacylglycerol $(r=-0.19, p=0.032)$ and ethnicity $(r=-0.34$, $p<0.001)$, and positively with LDL-cholesterol $(r=0.18$, $p=0.038)$. We found no correlation of baseline $\mathrm{Lp}$ (a) levels with sex $(r=0.08, p=0.389)$, fasting glucose $(r=-0.17$, $p=0.057)$ or fasting insulin levels $(r=-0.06, p=0.494)$. Participants of white origin had lower baseline $\mathrm{Lp}(\mathrm{a})$ levels than non-white participants: median $25.7 \mathrm{nmol} / 1$ (IQR 5.7-120.1) vs $122.0 \mathrm{nmol} / 1$ (IQR 34.0-214.6) $(p<0.001)$.

The diet resulted in a weight loss of $10.2 \mathrm{~kg}(95 \%$ CI 9.2, 11.3), which was equivalent to $9.9 \%$ (95\% CI 8.9, 10.8) of initial body weight. Both BMI and waist circumference decreased significantly ( $p<0.001$ for all). $\mathrm{HbA}_{1 \mathrm{c}}$ and fasting glucose levels decreased ( $p<0.001$ for both), indicating improved glycaemic control. Lipid variables also improved during the dietary intervention ( $p<0.01$ for all, Table 1$)$.

$\mathrm{Lp}$ (a) levels increased significantly from $40.9 \mathrm{nmol} / 1$ (IQR 13.9-159.5) to $55.9 \mathrm{nmol} / \mathrm{l}$ (IQR 23.0-201.1) $(p<0.001$, Table 1). Figure 1 shows a waterfall plot of the changes in $\mathrm{Lp}$ (a) per individual. Of the 131 participants, 49 showed an increase of over $21 \mathrm{nmol} / 1(10 \mathrm{mg} / \mathrm{dl})$, and only six 


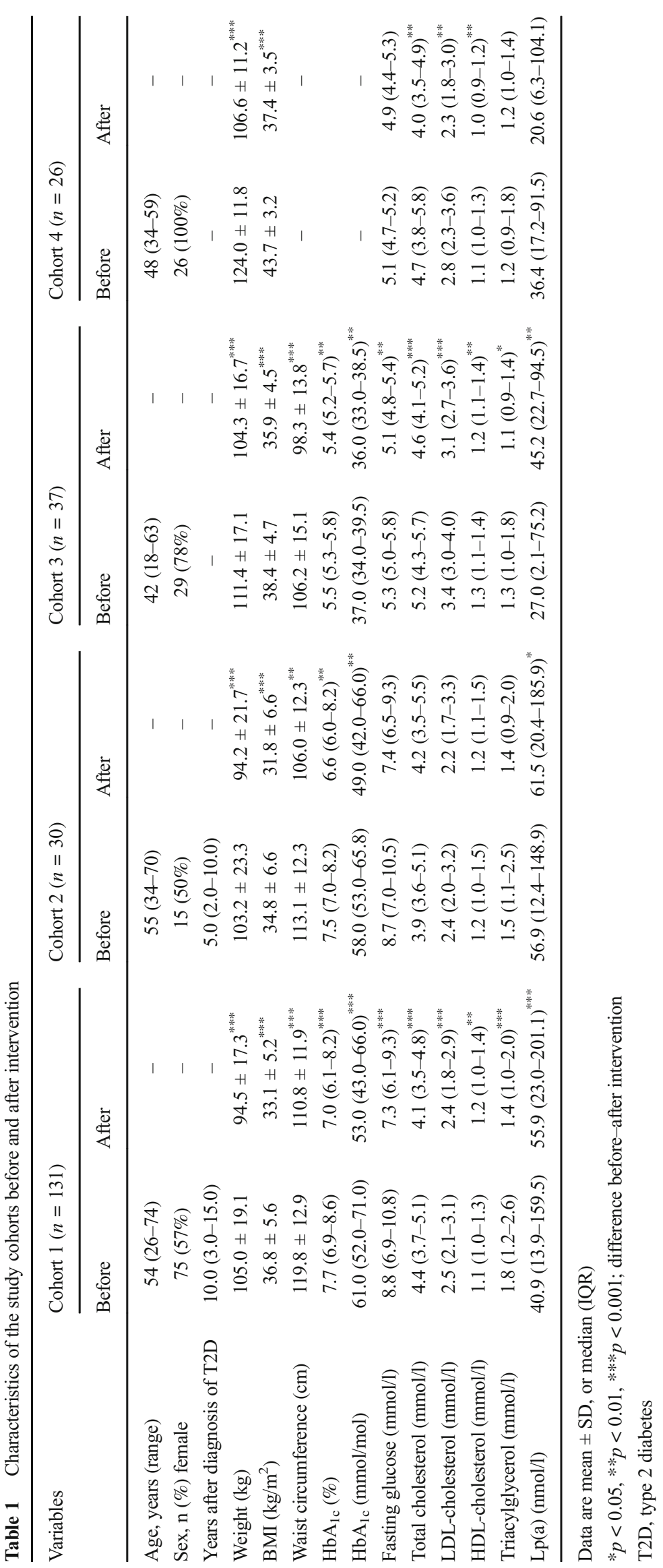




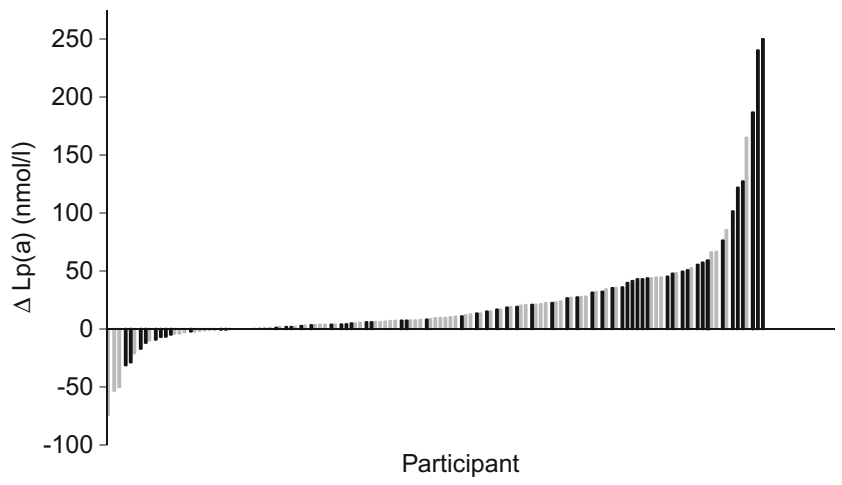

Fig. 1 Diet-induced changes in $L p(a)$ level per individual in cohort 1 $(n=131)$. Individual participants $(x$-axis) are arranged according to the diet-induced change in Lp(a) level. Grey bars, white participants; black bars, non-white participants

showed a decrease of $21 \mathrm{nmol} / 1$ or more $(10 \mathrm{mg} / \mathrm{dl})$. The median increase in $\mathrm{Lp}$ (a) levels in cohort 1 was $14.8 \mathrm{nmol} / 1$ (95\% CI 10.2, 20.6).

The change in $\mathrm{Lp}$ (a) correlated with baseline $\mathrm{Lp}$ (a) levels $(r=0.38, p<0.001)$ and with the change in fasting glucose $(r=-0.17, p=0.049)$ and LDL-cholesterol $(r=0.19$, $p=0.033$ ). The correlations with change in fasting glucose and LDL-cholesterol disappeared after correction for baseline $\mathrm{Lp}$ (a) levels. The change in $\mathrm{Lp}$ (a) did not correlate with sex $(r=-0.04, p=0.543)$ or change in weight $(r=-0.14$, $p=0.116)$. The change in $\mathrm{Lp}(\mathrm{a})$ also correlated with ethnicity (white vs non-white: $r=-0.17, p=0.048$ ), although this did not happen after correction for baseline Lp(a) levels. The $\mathrm{Lp}$ (a) response to the diet did not differ between white and non-white individuals in a repeated-measurements MANOVA $\left(\mathrm{F}_{(1 ; 129)}=0.199, p=0.656\right)$. In cohort 1,95 out of the 131 (73\%) participants used statins; the diet-induced change in Lp(a) levels was similar whether or not statins were used $\left(\mathrm{F}_{(1 ; 129)}=0.669, p=0.415\right)$.

Excluding two possible outliers with an increase Lp(a) level of $\geq 211 \mathrm{nmol} / 1$, the outcome was similar.

\section{Effect of Apo(a) isoform on diet-induced changes in Lp(a)} levels in cohort 1 Forty-three participants had an LMW and 88 an HMW Apo(a) isoform. As expected, baseline Lp(a) levels were significantly higher in the LMW than the HMW subgroup (148.8 nmol/1 [IQR 26.6-297.9] vs $30.6 \mathrm{nmol} / 1$ [IQR 6.5-119.4]; $p<0.001)$. Lp(a) levels increased during the dietary intervention to $182.7 \mathrm{nmol} / \mathrm{l}$ (IQR 37.3-327.5; $p<0.001)$ in the LMW subgroup and to $41.6 \mathrm{nmol} / 1$ (IQR 15.4-139.9; $p<0.001$ ) in the HMW subgroup (Fig. 2). The diet-induced effect on Lp(a) did not significantly differ between the LMW and the HMW subgroup $\left(\mathrm{F}_{(1 ; 129)}=1.68, p=0.197\right)$. The alteration in Lp(a) levels correlated strongly with baseline $\mathrm{Lp}$ (a) level in the HMW subgroup $(r=0.43, p<0.001)$ but not in the LMW subgroup $(r=0.24, p=0.118)$.

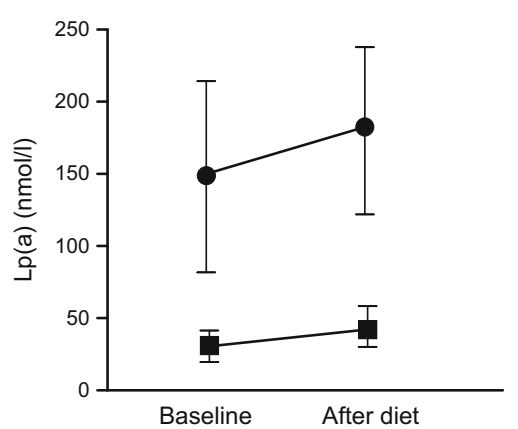

Fig. 2 The effect of the dietary intervention on $\mathrm{Lp}$ (a) level in the Apo(a) isoform subgroups in cohort 1. Medians and 95\% CIs of Lp(a) levels before and after the dietary intervention for the LMW Apo(a) isoform group (circles, $n=43$ ) and the HMW Apo(a) isoform group (squares, $n=88)$

Long-term effect Of the 131 participants in cohort 1, 69 consented to provide an additional blood sample 20 months after finishing the dietary intervention. This subgroup was older ( 55.6 vs 51.8 years, $p=0.016$ ), had a longer history of type 2 diabetes (12.2 vs 8.8 years, $p=0.017$ ) and had lost more weight during the intervention $(12.1 \mathrm{vs} 8.6 \mathrm{~kg}$, $p=0.001)$, but did not differ from the other participants in sex distribution, ethnicity, baseline $\mathrm{Lp}(\mathrm{a}), \mathrm{BMI}_{\mathrm{H}} \mathrm{HbA}_{1 \mathrm{c}}$ and LDL-cholesterol, nor in change in $\mathrm{Lp}$ (a) during the diet. In this subgroup, Lp(a) levels increased from $40.9 \mathrm{nmol} / 1$ (IQR 15.6-151.7) to $55.1 \mathrm{nmol} / \mathrm{l}$ (IQR 24.7-200.2) during the dietary intervention. Twenty months after the diet, patients had regained an average of $6.8 \pm 5.5 \mathrm{~kg}$ of body weight but were still $5.2 \pm 6.0 \mathrm{~kg}$ below baseline weight. Lp(a) levels decreased to $43.9 \mathrm{nmol} / \mathrm{l}$ (IQR 12.2-157.8), which was no longer statistically different from baseline levels $(p=0.050)$. Weight regain was not correlated with the decrease in $\mathrm{Lp}(\mathrm{a})$ levels from the end of the intervention to 20 months after the intervention $(r=-0.06, p=0.626)$.

Effect of weight loss on $\mathrm{Lp}$ (a) levels in secondary cohorts The characteristics of the cohorts $2-4$ at baseline and after the intervention are shown in Table 1. Cohort 2, consisting predominantly of obese patients with type 2 diabetes, showed effects of the diet similar to the primary cohort. Weight loss was $9.0 \mathrm{~kg}(95 \%$ CI 6.7, 11.3), or $8.5 \%$ (95\% CI 6.5, 10.6) of initial body weight, and both BMI and waist circumference decreased significantly ( $p<0.01$ for all). $\mathrm{HbA}_{1 \mathrm{c}}$ level also decreased $(p=0.001)$, but changes in fasting glucose and lipid variables (total cholesterol, triacylglycerol, LDL-cholesterol and HDL-cholesterol) did not reach statistical significance in this small group (Table 1). During dieting, Lp(a) increased from $56.9 \mathrm{nmol} / 1$ (IQR 12.4-148.9) to $61.5 \mathrm{nmol} / 1$ (IQR 20.4-185.9) ( $p=0.018$; Table 1). The median increase in $\mathrm{Lp}$ (a) was $13.5 \mathrm{nmol} / 1$ (95\% CI 2.3, 30.0).

In cohort 3 , which consisted of obese individuals without type 2 diabetes, the dietary intervention led to a weight loss of $7.1 \mathrm{~kg}(95 \%$ CI $6.3,8.0)$, or $6.5 \%$ (95\% CI $5.7,7.2)$ of 
initial body weight, and to significant reductions in BMI and waist circumference $(p<0.001$ for all). Although these participants did not have type 2 diabetes, $\mathrm{HbA}_{1 \mathrm{c}}$ and fasting glucose levels improved in this group $(p=0.002$ and $p=0.003$, respectively). In addition, lipid variables improved significantly ( $p<0.05$ for all). Lp(a) levels increased from $27.0 \mathrm{nmol} / 1$ (IQR 2.1-75.2) to $45.2 \mathrm{nmol} / \mathrm{l}$ (IQR 22.7-94.5) $(p=0.001$; Table 1). The median increase in $\mathrm{Lp}(\mathrm{a})$ was $11.9 \mathrm{nmol} / 1$ (95\% CI 5.7, 19.0).

Cohort 4 consisted of obese individuals without type 2 diabetes who underwent bariatric surgery and were followed up for 3 months. This intervention resulted in a weight loss of $17.4 \mathrm{~kg}(95 \%$ CI $15.0,19.8)$, or $14.0 \%$ (95\% CI 12.2, 15.7) of initial body weight $(p<0.001)$. During this period, most lipid variables improved significantly (Table 1). Lp(a) levels were lower after the intervention than before (falling from $36.4 \mathrm{nmol} / \mathrm{l}$ [IQR $17.2-91.5$ ] to $20.6 \mathrm{nmol} / 1$ [IQR 6.3-104.1]), but this result did not reach statistical significance in this small group (Table 1). The median difference in $\mathrm{Lp}$ (a) level was $-7.0 \mathrm{nmol} / 1$ (95\% CI -18.8, 5.3).

Figure 3 summarises the results obtained for the four independent cohorts. The relationship between weight loss and increase in $\mathrm{Lp}$ (a) levels was similar for the first three cohorts. When cohorts 1-3 were considered together, the increase in $\mathrm{Lp}$ (a) correlated with the diet-induced weight loss ( $n=198, r=-0.18, p=0.012$ ). This relationship was not observed for cohort 4 , which consisted of individuals who lost weight after bariatric surgery.

\section{Discussion}

Our data show that diet-induced weight loss increased Lp(a) levels in overweight and obese individuals irrespective of the presence or absence of type 2 diabetes. Repeated sampling in healthy control participants at an interval of 2-6 months showed that the increase in $L p(a)$ levels was not explained by general environmental changes over time or by assay

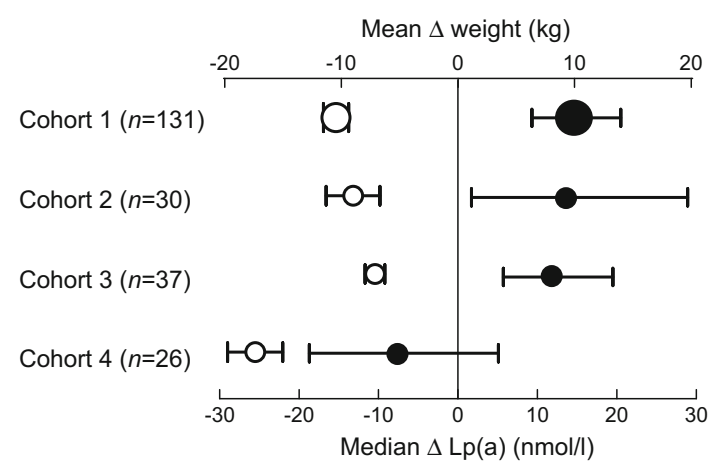

Fig. $3 \Delta \mathrm{Lp}(\mathrm{a})$ and $\Delta$ weight in the four independent study cohorts. Means and $95 \%$ CI for $\Delta$ weight (white circles) and medians with $95 \%$ CIs for $\Delta \mathrm{Lp}$ (a) (black circles) in the four cohorts. The size of the symbols reflects the number of participants artefacts. In patients with type 2 diabetes, the extent of the increase in $\mathrm{Lp}$ (a) was mainly determined by baseline $\mathrm{Lp}(\mathrm{a})$ level, with the highest increase seen in individuals with the highest baseline levels. This effect on $\mathrm{Lp}(\mathrm{a})$ was independent of the Apo(a) isoform. Such an increase in $\mathrm{Lp}(\mathrm{a})$ levels was not observed in individuals who underwent bariatric surgery, suggesting that weight loss per se does not increase Lp(a) levels.

Previous studies have not shown a change in $L p(a)$ levels in obese adults after various dietary interventions aimed at weight loss [21-23]. In these studies, weight-reducing drugs and diets different from ours were tested, and patients with type 2 diabetes were not included. One study reported a decrease in $\mathrm{Lp}$ (a) levels in obese children [20]. This discrepancy in relation to our study may be explained by different age-related hormonal states or by differences in dietary composition. The type and content of fat in the diet may be an important determinant of the dietary effect on $L p(a)$ levels. An increased intake of total and saturated fat has been found to decrease $L p(a)$ levels, while an increased intake of monounsaturated fatty acids tended to increase $\mathrm{Lp}$ (a) levels in healthy individuals and those with metabolic dysregulation [29-31]. Faghihnia et al [30] have suggested that a low-fat diet results in an increase in $\mathrm{Lp}$ (a) levels that may be due to an altered metabolism of $\mathrm{Lp}$ (a) particles. The dietary interventions used in our cohorts 1-3 were all based on a low intake of total and saturated fat, while no specific dietary restrictions were prescribed for the participants in the cohort who underwent bariatric surgery. We previously reported that, in a random subset of participants in cohort 1 , our dietary intervention lowered plasma levels of the soluble form of the LDL receptor relative with 11 ligand-binding repeats (sLR11) [32]. However, diet-induced changes in sLR11 and Lp(a) levels did not correlate with each other $(r=-0.07$, $p=0.635)$. In participants in cohort 1 from whom blood samples were available at 20 months of follow-up, Lp(a) levels had almost returned to baseline values, whereas the initial weight reduction was only partially reversed by weight regain. This suggests that the increase in $L p(a)$ levels was an acute effect of the diet. Unfortunately, we do not have information about the diet during the prolonged follow-up. Future studies on the effect on $\mathrm{Lp}(\mathrm{a})$ of weight-reducing diets with different fat contents in obese patients with and without type 2 diabetes are warranted.

High Lp(a) levels have consistently been associated with an increased risk of coronary heart disease [6,9], and results from genetic studies indicate a causal association between high Lp(a) levels and CVD [17, 33, 34]. The risk of CVD associated with high $\mathrm{Lp}$ (a) levels is notably higher in individuals with than without type 2 diabetes [18]. The dose-response relationship of $\mathrm{Lp}(\mathrm{a})$ levels with CVD risk has been shown to be curvilinear in shape, with no evidence of a threshold [35]. This suggests that the increase in $L p(a)$ 
levels induced by weight loss dieting observed in our study might increase the risk of CVD. This could potentially reduce the beneficial cardiometabolic effects that result from the improvement in conventional CVD risk factors after diet-induced weight loss. In the Look AHEAD (Action for Health in Diabetes) study (NCT00017953), the incidence of CVD was not reduced by a low-energy, low-fat diet and physical activity in patients with type 2 diabetes after 10 years of follow-up, despite an improvement in conventional risk factors for CVD [5]. Hypothetically, a parallel increase in Lp(a) levels could be one of the explanations why CVD events were not reduced by this lifestyle change. However, effects on $L p(a)$ levels were not reported in the Look AHEAD trial. Randomised clinical trials addressing the effect of alterations in $\mathrm{Lp}(\mathrm{a})$ levels with lifestyle changes or medication on hard clinical endpoints or CVD risk are needed. Recently, the short-term efficacy and safety of two specific Lp(a)-lowering agents has been reported [36]. Long-term effects on cardiovascular endpoints are awaited.

In participants who underwent bariatric surgery, weight loss was not accompanied by an increase in $L p(a)$ level. Two previous studies have shown that bariatric surgery-induced weight loss in obese individuals was accompanied by a decrease in $\mathrm{Lp}$ (a) levels [37, 38], whereas another study found no significant effect [39]. The effects of bariatric surgery on bile acid flow and signalling, inflammation, release of gastrointestinal hormones, the gut microbiome and the wound healing processes may all have had an impact on $\mathrm{Lp}(\mathrm{a})$, resulting in the absence of a weight loss-induced increase in Lp(a) levels [40-44].

The baseline $\mathrm{Lp}$ (a) levels in our two cohorts with type 2 diabetes (cohorts 1 and 2) were relatively high compared with the two cohorts without type 2 diabetes (cohorts 3 and 4), whereas in the Women's Health Study and Copenhagen City Heart Study the Lp(a) levels in participants with diabetes were significantly lower than the $\mathrm{Lp}$ (a) levels in the control participants $[45,46]$. Non-white individuals, in particular those of South-Asian ancestry, display markedly higher Lp(a) levels than white individuals [47-49], and are over-represented in our cohorts with type 2 diabetes. The change in $\mathrm{Lp}(\mathrm{a})$ during the diet was correlated with ethnicity. However, in the repeated-measurements analysis we found no difference between the white and non-white populations in $\Delta \mathrm{Lp}(\mathrm{a})$. This suggests that non-white individuals have higher baseline $\mathrm{Lp}$ (a) levels, and therefore show the highest absolute change in $\mathrm{Lp}(\mathrm{a})$ levels on dieting, but that the relative change is similar to that in white individuals.

The strengths of this study are its prospective design and the use of four independent cohorts for investigating the effect of weight loss on $\operatorname{Lp}(\mathrm{a})$, which more than doubled the total number of participants who have so far been studied in relation to this topic. Our study is descriptive in nature. Future studies should clarify the mechanisms underlying the increase in Lp(a) levels on diet-induced weight loss, as well as the consequence of weight loss for the functionality of $L p(a)$. As all the participants had been referred to a tertiary centre, our findings may not be applicable to the entire population of overweight and obese patients with or without type 2 diabetes. We found that the effect of diet-induced weight loss on $L p(a)$ levels occurred irrespective of the presence or absence of type 2 diabetes. However, some of the individuals in cohorts 3 and 4 may have had impaired glucose tolerance, since the classification was based on fasting glucose level and not on an oral glucose tolerance test. Finally, a long-term follow-up study is required to determine whether elevated $\mathrm{Lp}$ (a) levels after weight loss dieting affect the incidence of CVD in obese patients with and without type 2 diabetes.

In conclusion, $L p(a)$ levels increased significantly in obese individuals with and without type 2 diabetes during diet-induced weight loss, but not in individuals who underwent bariatric surgery. This may hypothetically reduce the beneficial cardiometabolic effects of a diet-induced weight loss. Therefore, $\mathrm{Lp}(\mathrm{a})$ may be an additional target in overweight and obese individuals on a energy-restricted diet to reduce the risk of CVD. Long-term follow-up studies are required to establish whether adding a specific $\mathrm{Lp}$ (a)-lowering agent to a dietary intervention will improve long-term CVD outcome in obese individuals with and without type 2 diabetes.

Acknowledgements Part of the data was presented at the 2016 Annual Dutch Diabetes Research Meeting in Oosterbeek, the Netherlands, and at the EAS 2016 Lp(a) satellite meeting in Innsbruck, Austria.

Data availability statement All data are available on request from the authors.

Funding This work was supported by the Erasmus Medical Center within the funding programme Zorgonderzoek Erasmus MC, ID 2008-8303, and the Diabetes Foundation, the Netherlands, ID 2013.30.1684. The funding body had no involvement in the design of the trial, in the collection, analysis and interpretation of the data, or in the decision to submit the manuscript for publication.

Duality of interest The authors declare that there is no duality of interest associated with this manuscript.

Contribution statement $\mathrm{KAB}$ participated in the design of the study, recruited all the participants in cohorts 1 and 2, collected and analysed data, and wrote the manuscript. RY participated in the design of the study, analysed data, contributed to the writing and edited the manuscript. AJMV participated in the design of the study, reviewed/edited the manuscript and contributed significantly to the discussion. JT and FPL contributed to the acquisition, analysis and interpretation of data and reviewed the manuscript. EFvR and VLW recruited the individuals in cohort 3, collected data, participated in the design of the study and reviewed/edited the manuscript. MAL and HP recruited the individuals in cohort 4 , collected data, participated in the design of the study and reviewed/edited the manuscript. RT conducted the data analysis and contributed to the design of the study and the writing of the manuscript. 
GE, FK and JERvL participated in the design of the study, reviewed and edited the manuscript, and contributed significantly to the discussion. EJGS and MTM contributed substantially to the conception and design, the analysis and interpretation of data, and critical revision of the paper for important intellectual content. All authors approved the final version of the manuscript. The guarantors of the manuscript are MTM and EJGS.

Open Access This article is distributed under the terms of the Creative Commons Attribution 4.0 International License (http:// creativecommons.org/licenses/by/4.0/), which permits unrestricted use, distribution, and reproduction in any medium, provided you give appropriate credit to the original author(s) and the source, provide a link to the Creative Commons license, and indicate if changes were made.

\section{References}

1. Costanzo P, Cleland JG, Pellicori P et al (2015) The obesity paradox in type 2 diabetes mellitus: relationship of body mass index to prognosis: a cohort study. Ann Intern Med 162:610-618

2. Joseph JJ, Golden SH (2014) Type 2 diabetes and cardiovascular disease: what next? Curr Opin Endocrinol Diabetes Obes 21:109120

3. Global Burden of Metabolic Risk Factors for Chronic Diseases Collaboration, Lu Y, Hajifathalian K et al (2014) Metabolic mediators of the effects of body-mass index, overweight, and obesity on coronary heart disease and stroke: a pooled analysis of 97 prospective cohorts with 1.8 million participants. Lancet 383:970-983

4. Anderson JW, Kendall CW, Jenkins DJ (2003) Importance of weight management in type 2 diabetes: review with meta-analysis of clinical studies. J Am Coll Nutr 22:331-339

5. Wing RR, Bolin P, Brancati FL et al (2013) Cardiovascular effects of intensive lifestyle intervention in type 2 diabetes. N Engl J Med 369:145-154

6. Bennet A, Di Angelantonio E, Erqou S et al (2008) Lipoprotein(a) levels and risk of future coronary heart disease: large-scale prospective data. Arch Intern Med 168:598-608

7. Kamstrup PR (2010) Lipoprotein(a) and ischemic heart disease - a causal association? A review. Atherosclerosis 211:15-23

8. Kamstrup PR, Benn M, Tybjaerg-Hansen A, Nordestgaard BG (2008) Extreme lipoprotein(a) levels and risk of myocardial infarction in the general population: the Copenhagen City Heart Study. Circulation 117:176-184

9. Hopewell JC, Seedorf U, Farrall M et al (2014) Impact of lipoprotein(a) levels and apolipoprotein(a) isoform size on risk of coronary heart disease. J Intern Med 276:260-268

10. Hiraga T, Kobayashi T, Okubo M et al (1995) Prospective study of lipoprotein(a) as a risk factor for atherosclerotic cardiovascular disease in patients with diabetes. Diabetes Care 18:241-244

11. James RW, Boemi M, Sirolla C, Amadio L, Fumelli P, Pometta D (1995) Lipoprotein (a) and vascular disease in diabetic patients. Diabetologia 38:711-714

12. Emerging Risk Factors Collaboration, Erqou S, Kaptoge $\mathrm{S}$ et al (2009) Lipoprotein(a) concentration and the risk of coronary heart disease, stroke, and nonvascular mortality. JAMA 302:412-423

13. Boerwinkle E, Leffert CC, Lin J, Lackner C, Chiesa G, Hobbs HH (1992) Apolipoprotein(a) gene accounts for greater than $90 \%$ of the variation in plasma lipoprotein(a) concentrations. J Clin Invest 90: $52-60$

14. Kraft HG, Kochl S, Menzel HJ, Sandholzer C, Utermann G (1992) The apolipoprotein (a) gene: a transcribed hypervariable locus controlling plasma lipoprotein (a) concentration. Hum Genet 90:220 230
15. Lackner C, Cohen JC, Hobbs HH (1993) Molecular definition of the extreme size polymorphism in apolipoprotein(a). Hum Mol Genet 2:933-940

16. Scanu AM, Fless GM (1990) Lipoprotein (a). Heterogeneity and biological relevance. J Clin Invest 85:1709-1715

17. Kamstrup PR, Tybjaerg-Hansen A, Steffensen R, Nordestgaard BG (2009) Genetically elevated lipoprotein(a) and increased risk of myocardial infarction. JAMA 301:2331-2339

18. Waldeyer C, Makarova N, Zeller T et al (2017) Lipoprotein(a) and the risk of cardiovascular disease in the European population results from the BiomarCaRE consortium. Eur Heart J DOI:10. 1093/eurheartj/ehx166

19. Kronenberg F, Utermann G (2013) Lipoprotein(a): resurrected by genetics. J Intern Med 273:6-30

20. Brandstatter A, Lingenhel A, Zwiauer K, Strobl W, Kronenberg F (2009) Decrease of Lp(a) during weight reduction in obese children is modified by the apo(a) kringle-IV copy number variation. Int $\mathrm{J}$ Obes 33:1136-1142

21. Corsetti JP, Sterry JA, Sparks JD, Sparks CE, Weintraub M (1991) Effect of weight loss on serum lipoprotein(a) concentrations in an obese population. Clin Chem 37:1191-1195

22. Kiortsis DN, Tzotzas T, Giral P et al (2001) Changes in lipoprotein(a) levels and hormonal correlations during a weight reduction program. Nutr Metab Cardiovasc Dis 11:153-157

23. Yamashita T, Sasahara T, Pomeroy SE, Collier G, Nestel PJ (1998) Arterial compliance, blood pressure, plasma leptin, and plasma lipids in women are improved with weight reduction equally with a meatbased diet and a plant-based diet. Metabolism 47:1308-1314

24. Berk KA, Buijks H, Ozcan B, Van't Spijker A, Busschbach JJ, Sijbrands EJ (2012) The Prevention Of WEight Regain in diabetes type 2 (POWER) study: the effectiveness of adding a combined psychological intervention to a very low calorie diet, design and pilot data of a randomized controlled trial. BMC Public Health 12:1026

25. Berk KA, Oudshoorn TP, Verhoeven AJM et al (2016) Dietinduced weight loss and markers of endothelial dysfunction and inflammation in treated patients with type 2 diabetes. Clin Nutr ESPEN 15:101-106

26. Mujibul Haq AM, M Giasuddin AS, Huque MM (2011) Serum total homocysteine and lipoprotein (a) levels in acute myocardial infarction and their response to treatment with vitamins. J Coll Physicians Surg Pak 21:266-270

27. Vongpromek R, Bos S, Ten Kate GJ et al (2015) Lipoprotein(a) levels are associated with aortic valve calcification in asymptomatic patients with familial hypercholesterolaemia. J Intern Med 278: 166-173

28. Kronenberg F, Kuen E, Ritz E et al (2000) Lipoprotein(a) serum concentrations and apolipoprotein(a) phenotypes in mild and moderate renal failure. J Am Soc Nephrol 11:105-115

29. Clevidence BA, Judd JT, Schaefer EJ et al (1997) Plasma lipoprotein (a) levels in men and women consuming diets enriched in saturated, cis-, or trans-monounsaturated fatty acids. Arterioscler Thromb Vasc Biol 17:1657-1661

30. Faghihnia N, Tsimikas S, Miller ER, Witztum JL, Krauss RM (2010) Changes in lipoprotein(a), oxidized phospholipids, and LDL subclasses with a low-fat high-carbohydrate diet. J Lipid Res 51:3324-3330

31. Berglund L, Lefevre M, Ginsberg HN et al (2007) Comparison of monounsaturated fat with carbohydrates as a replacement for saturated fat in subjects with a high metabolic risk profile: studies in the fasting and postprandial states. Am J Clin Nutr 86:1611-1620

32. Berk KA, Vongpromek R, Jiang M et al (2016b) Levels of the soluble LDL receptor-relative LR11 decrease in overweight individuals with type 2 diabetes upon diet-induced weight loss. Atherosclerosis 254:67-72 
33. Clarke R, Peden JF, Hopewell JC et al (2009) Genetic variants associated with $\mathrm{Lp}$ (a) lipoprotein level and coronary disease. $\mathrm{N}$ Engl J Med 361:2518-2528

34. Tregouet DA, Konig IR, Erdmann J et al (2009) Genome-wide haplotype association study identifies the SLC22A3-LPAL2-LPA gene cluster as a risk locus for coronary artery disease. Nat Genet 41:283-285

35. Nordestgaard BG, Chapman MJ, Ray K et al (2010) Lipoprotein(a) as a cardiovascular risk factor: current status. Eur Heart J 31:2844-2853

36. Viney NJ, van Capelleveen JC, Geary RS et al (2016) Antisense oligonucleotides targeting apolipoprotein(a) in people with raised lipoprotein(a): two randomised, double-blind, placebo-controlled, dose-ranging trials. Lancet 388:2239-2253

37. Williams DB, Hagedorn JC, Lawson EH et al (2007) Gastric bypass reduces biochemical cardiac risk factors. Surg Obes Relat Dis 3:8-13

38. Ram E, Vishne T, Magazanik A et al (2007) Changes in blood lipid levels following silastic ring vertical gastroplasty. Obes Surg 17: 1292-1296

39. Woodard GA, Peraza J, Bravo S, Toplosky L, Hernandez-Boussard T, Morton JM (2010) One year improvements in cardiovascular risk factors: a comparative trial of laparoscopic Roux-en-Y gastric bypass vs. adjustable gastric banding. Obes Surg 20:578-582

40. Chennamsetty I, Claudel T, Kostner KM et al (2011) Farnesoid X receptor represses hepatic human APOA gene expression. J Clin Invest 121:3724-3734

41. Huang M, Gong Y, Grondolsky J, Hoover-Plow J (2014) Lp(a)/ apo(a) modulate MMP-9 activation and neutrophil cytokines in vivo in inflammation to regulate leukocyte recruitment. Am J Pathol 184:1503-1517

42. Nielsen LB, Stender S, Kjeldsen K, Nordestgaard BG (1996) Specific accumulation of lipoprotein(a) in balloon-injured rabbit aorta in vivo. Circ Res 78:615-626

43. von Zychlinski A, Kleffmann T, Williams MJ, McCormick SP (2011) Proteomics of lipoprotein(a) identifies a protein complement associated with response to wounding. J Proteome 74:28812891

44. Yano Y, Shimokawa K, Okada Y, Noma A (1997) Immunolocalization of lipoprotein(a) in wounded tissues. J Histochem Cytochem 45:559 568

45. Kamstrup PR, Nordestgaard BG (2013) Lipoprotein(a) concentrations, isoform size, and risk of type 2 diabetes: a Mendelian randomisation study. Lancet Diabetes Endocrinol 1:220-227

46. Mora S, Kamstrup PR, Rifai N, Nordestgaard BG, Buring JE, Ridker PM (2010) Lipoprotein(a) and risk of type 2 diabetes. Clin Chem 56:1252-1260

47. Enkhmaa B, Anuurad E, Berglund L (2016) Lipoprotein (a): impact by ethnicity and environmental and medical conditions. J Lipid Res 57:1111-1125

48. Banerjee D, Wong EC, Shin J, Fortmann SP, Palaniappan L (2011) Racial and ethnic variation in lipoprotein (a) levels among Asian Indian and Chinese patients. J Lipid 2011:291954

49. Hoogeveen RC, Gambhir JK, Gambhir DS et al (2001) Evaluation of $\mathrm{Lp}[\mathrm{a}]$ and other independent risk factors for CHD in Asian Indians and their USA counterparts. J Lipid Res 42:631-638 